\title{
Coexistence de Nématodes Trichostrongyloides chez des Marsupiaux de Guyane
}

\author{
par O.-T. DIAW \\ Laboratoire de Zoologie (Vers), associé au C.N.R.S., \\ Muséum national d'Histoire naturelle, 43, rue Cuvier, F 75231 Paris Cedex 05
}

\section{Résumé.}

Analyse de l'helminthofaune de deux Didelphis marsupialis comparée à celle faite par Durette-Desset, 1974, chez un Metachirops opossum de la même région.

Bien que l'équilibre général soit assez comparable, les différences entre les deux Marsupiaux sont sensibles, l'espèce dominante, en particulier, n'est pas la même. La localisation des espèces communes ou des espèces vicariantes est stable. (Contrairement aux premières notions acquises à ce sujet, il apparaît que la faune est constituée d'une seule et même lignée, à l'exception des Moennigia, qui seraient des captures provenant des Xenarthes).

\section{Sumimary.}

Coexistence of Nematodes Trichostrongyloidea in Marsupials from French Guyana.

Analysis of the helminthofauna of two Didelphis marsupialis, compared to that of a Metachirops opossum from the same area, studied by Durette-Desset, 1974. Except for their similarities in the general repartition of the species, the two Marsupials are easily differentiated: the dominant species is not the same. The location of common or \&vicariant » species is stable. In opposition to former notions, the fauna appears to be composed of but one lineage, except for Moennigia, which would appear as captures from Xenarthes. 
Les problèmes posés par la coexistence de plusieurs espèces dans l'intestin d'un hôte ont été abordés à plusieurs reprises, chez divers groupes de Nématodes, en particulier chez les Oxyures (voir A. Petter, 1966) et chez les Trichostrongyloidea (voir Durette-Desset, 1971).

Ayant eu l'occasion dans un article précédent (cf. Diaw, 1976) d'étudier du point de vue morphologique la faune intestinale de deux Marsupiaux originaires de Guyane, il nous a paru intéressant de compléter ce travail en abordant les problèmes écologiques et phylétiques posés par la présence de telles faunes.

Après avoir comparé nos résultats avec ceux trouvés chez un Metachirops opossum (cf. Durette-Desset, 1974), nous émettrons quelques hypothèses sur la formation de l'helminthofaune (Trichostrongyloidea) chez les Marsupiaux néo-tropicaux.

\section{I. Étude des Trichostrongyloidea parasites de deux Didelphis marsupialis}

\section{Exposé des résultats :}

Lors de l'autopsie, l'intestin du Didelphis $\mathrm{n}^{\circ} 327$ BA a été divisé en huit parties sensiblement égales, de l'estomac au cæcum, celui du Didelphis $\mathrm{n}^{\circ} 57 \mathrm{HA}$ en quatre parties, du duodénum au cæcum.

a) NOMBRE D'ESPÈCES TROUVÉES :

Chez le 327 BA, les espèces suivantes ont été trouvées: Travassostrongylus callis (Travassos, 1914); T. orloffi Travassos, 1935; Viannaia viannai Travassos, 1914 ; Moennigia dessetae Diaw, 1976. Chez le $57 \mathrm{BA}$, outre les quatre espèces précédentes, on trouve également Travassostrongylus tourei Diaw, 1976 et Hoineffia cayennensis Diaw, 1976. La faune du 327 BA se compose de 175 spécimens et celle du 57 HA de 1542 spécimens.

TABleAu I. - Didelphis marsupialis 327 BA

\begin{tabular}{|c|c|c|c|c|c|}
\hline $\begin{array}{c}\text { Espèces } \\
\mathrm{N}^{\circ} \text { Portion }\end{array}$ & $\begin{array}{c}\text { Moennigia } \\
\text { dessetae }\end{array}$ & $\begin{array}{l}\text { Viannaia } \\
\text { viannai }\end{array}$ & $\begin{array}{l}\text { Travasso- } \\
\text { strongylus } \\
\text { orloffi }\end{array}$ & T. callis & Total \\
\hline I $\ldots \ldots \ldots \ldots \ldots$ & 3 & 60 & 18 & 11 & 92 \\
\hline II $\ldots \ldots \ldots \ldots \ldots$ & & 17 & 27 & 22 & 66 \\
\hline III $\ldots \ldots \ldots \ldots \ldots$ & & 6 & 6 & 3 & 15 \\
\hline IV $\ldots \ldots \ldots \ldots \ldots$ & & 2 & & & 2 \\
\hline Total $\ldots \ldots \ldots \ldots$ & 3 & 85 & 51 & 36 & 175 \\
\hline Pourcentages $\ldots \ldots$ & & 48,7 & 29 & 20,7 & \\
\hline
\end{tabular}




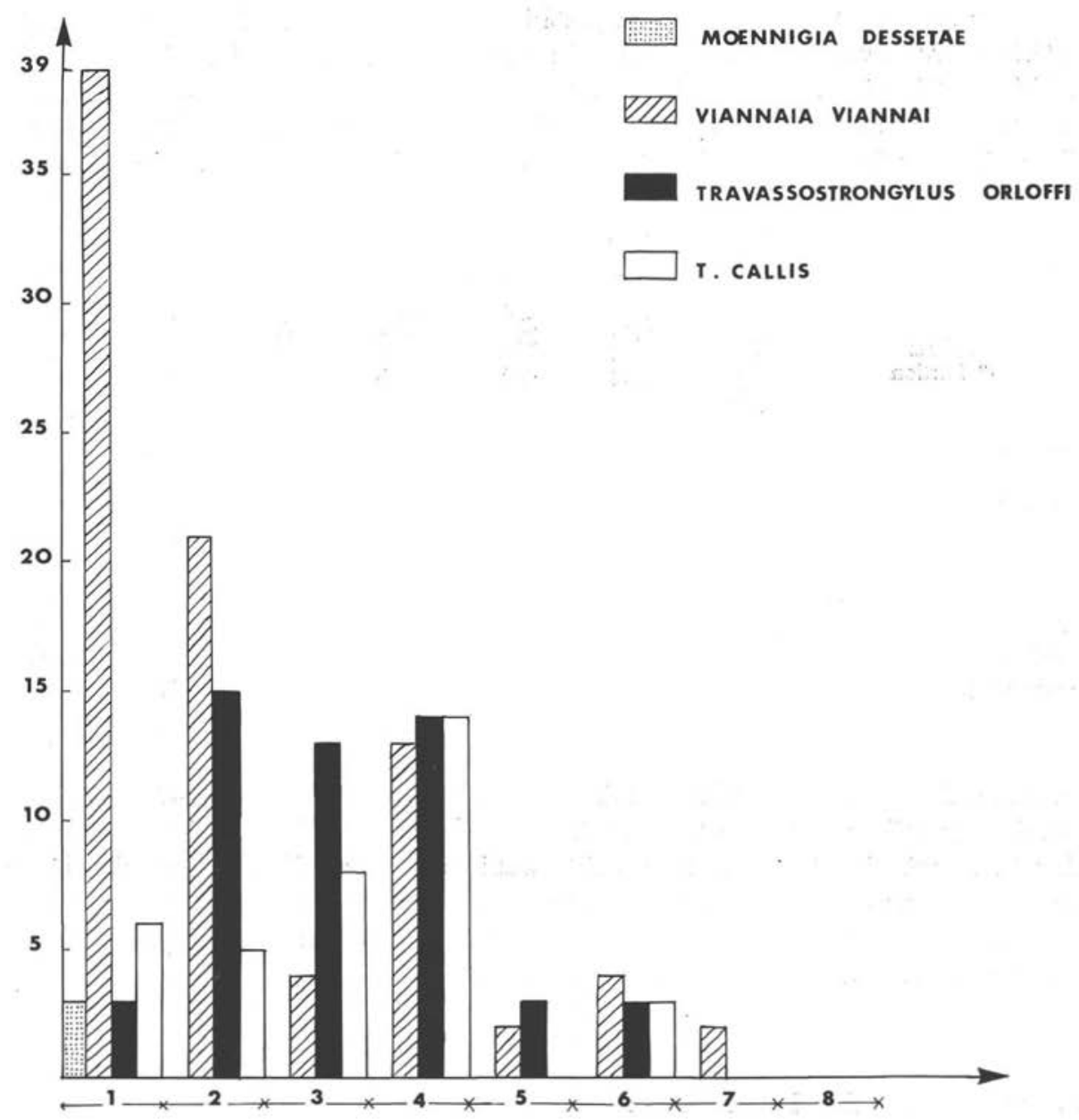

FIG. 1. - Répartition des différentes espèces de Trichostrongyloidea dans l'intestin d'un Didelphis marsupialis (327 BA) originaire de Guyane française. L'intestin est divisé en huit segments. Les chiffres en abscisse indiquent le numéro du segment depuis l'estomac jusqu'au cæcum. La totalité des individus de chaque espèce trouvée dans chaque segment est portée en ordonnée.

b) FréQuence RELATIVE DES ESPÈces :

Localisation dans l'intestin. Chez le Didelphis 327 BA, Viannaia viannai est l'espèce la plus abondante ( 85 spécimens) ; elle est présente dans toutes les parties de l'intestin et pour les 9/10 dans le quart antérieur. $T$. orloffi et $T$. callis, moins abondants que V. viannai (51 spécimens pour la première espèce, 36 pour la seconde), sont répartis 
dans tout l'intestin, sauf dans le quart postérieur, et sont plus abondants dans la partie antérieure. $M$. dessetae est une espèce très rare (3 spécimens) localisée dans la partie antérieure. Chez le $57 \mathrm{HA}, V$. viannai est l'espèce la plus abondante (1 410 spécimens) et se trouve dans toutes les parties de l'intestin, dont les 9/10 dans la moitié antérieure. $T$. orloffi, $T$. callis et $T$. tourei sont nettement moins abondants que $V$. viannai, repré-

TABleaU II. - Didelphis marsupialis 57 HA

\begin{tabular}{|c|c|c|c|c|c|c|c|}
\hline $\begin{array}{l}\text { Espèces } \\
\mathrm{N}^{\circ} \text { Portion }\end{array}$ & 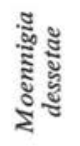 & 芯 & 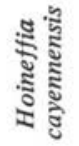 & 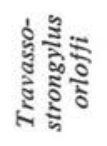 & 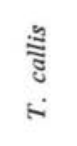 & $\begin{array}{l}\mathrm{a} \\
\stackrel{3}{3}\end{array}$ & छूँ \\
\hline Estomac $\ldots \ldots \ldots \ldots \ldots$ & 5 & 108 & & 3 & 12 & & 128 \\
\hline I $\ldots \ldots \ldots \ldots \ldots \ldots \ldots$ & & 559 & & 16 & 5 & 9 & 589 \\
\hline II $\ldots \ldots \ldots \ldots \ldots \ldots \ldots$ & & 624 & & 28 & 17 & 19 & 688 \\
\hline III $\ldots \ldots \ldots \ldots \ldots \ldots$ & & 112 & & & & & 123 \\
\hline IV $\ldots \ldots \ldots \ldots \ldots \ldots$ & & 7 & 7 & & & 11 & 14 \\
\hline Total $\ldots \ldots \ldots \ldots \ldots \ldots$ & 5 & 1410 & 7 & 47 & 34 & 39 & 1542 \\
\hline Pourcentages $\ldots \ldots \ldots$. & & 91,4 & & 3 & 2,2 & 2,5 & \\
\hline
\end{tabular}

sentés sensiblement par le même nombre de spécimens (respectivement 47, 34 et 39). Les deux premières espèces se trouvent dans l'estomac et la moitié antérieure de l'intestin ; $T$. tourei, absent de l'estomac, existe aussi dans la moitié antérieure de l'intestin, mais se trouve en outre dans la partie III. H. cayennensis est peu représenté (7) et n'existe que dans le quart postérieur. $M$. dessetae est très rare ( 5 spécimens) et n'existe que dans la partie antérieure (fig. 1, 2 et tableaux I, II).

\section{Comparaison des deux faunes :}

a) Différences :

La faune du $57 \mathrm{HA}$ est plus abondante (1 542 spécimens) que celle du $327 \mathrm{BA}$ (175 spécimens) ; elle possède également plus d'espèces (6) alors que le $327 \mathrm{BA}$ n'en possède que 4 .

\section{b) Ressemblances :}

Quatre espèces sont communes aux deux Didelphis : $T$. callis, $T$. orloffi, $V$. viannai et $M$. dessetae. Leur localisation dans l'intestin est identique pour chacun des deux individus-hôtes. 


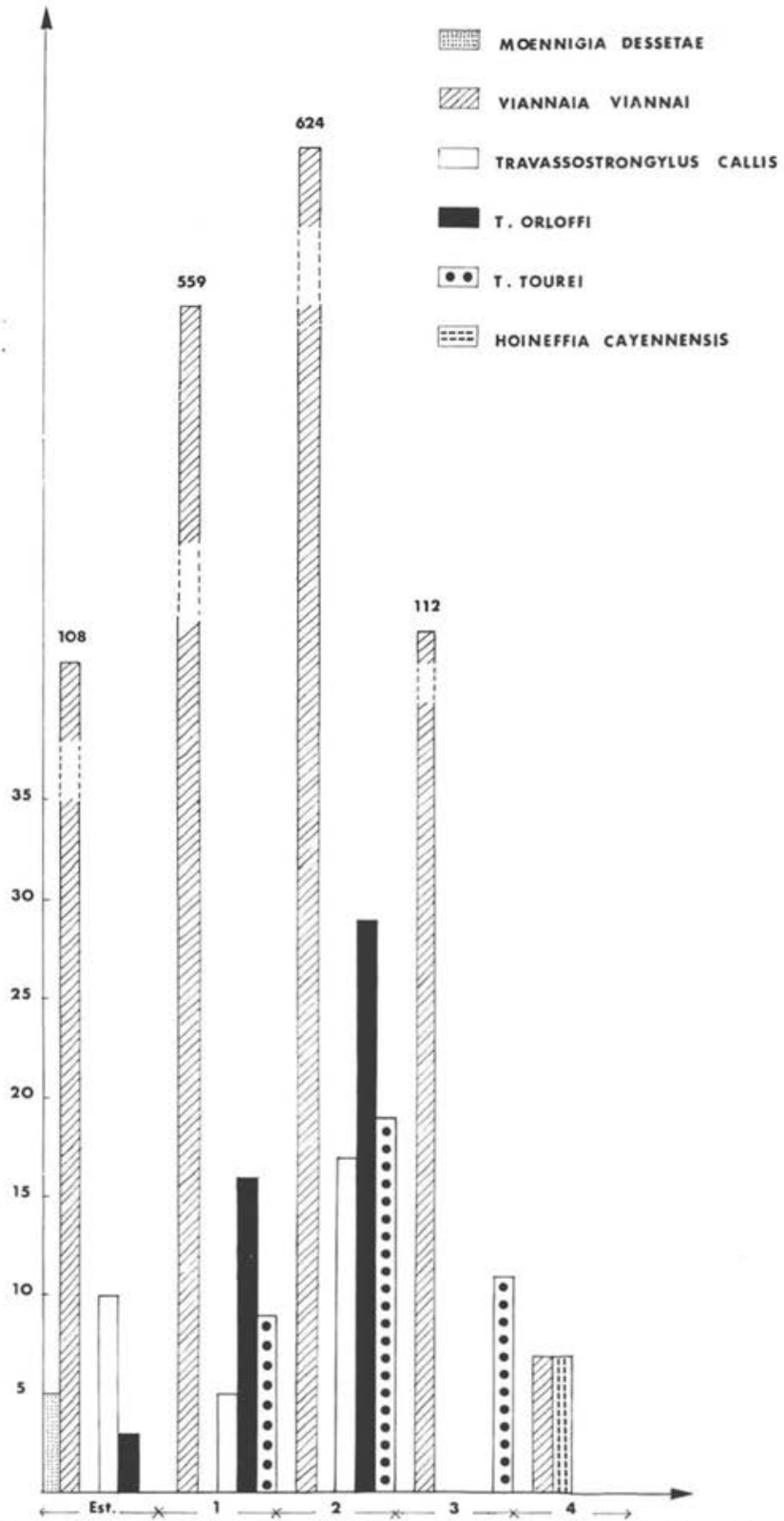

FIG. 2. - Répartition des différentes espèces de Trichostrongyloidea dans l'intestin d'un Didelphis marsupialis (57 HA) originaire de Guyane française. L'intestin est divisé en quatre segments. Les chiffres en abscisse indiquent le numéro du segment depuis l'estomac inclus jusqu'au cæcum. La totalité des individus de chaque espèce trouvée dans chaque segment est portée en ordonnée. 


\section{Comparaison avec l'helminthofaune \\ d'un autre Marsupial de la même famille et de la même région : Metachirops opossum}

\section{Rappel des résultats.}

L'intestin du Metachirops a été divisé en trois parties sub-égales du duodénum au cæcum. La faune comprend 67 spécimens répartis en quatre espèces : T. paraquintus Durette-Desset, 1974, V. viannai, V. metachirops Durette-Desset, 1974 et $M$. dessetae Diaw, 1976. $T$. paraquintus est l'espèce la plus abondante (50 spécimens) et se trouve dans tout l'intestin, répartie de façon équivalente dans les trois parties. $V$. viannai (11 spécimens) se trouve également dans tout l'intestin avec une répartition homogène. $V$. metachirops peu abondante (3 spécimens) n'est présente que dans la partie moyenne. Enfin, $M$. dessetae est représentée par trois spécimens et ne se trouve que dans la partie antérieure (fig. 3 et tableau III).

TABLEAU III. - Metachirops opossum

\begin{tabular}{lccccc}
\hline $\begin{array}{c}\text { Espèces } \\
\mathrm{N}^{\circ} \text { Portion }\end{array}$ & $\begin{array}{c}\text { Travasso- } \\
\text { strongylus } \\
\text { paraquintus }\end{array}$ & $\begin{array}{c}\text { Viannaia } \\
\text { viannai }\end{array}$ & $\begin{array}{c}\text { Viannaia } \\
\text { metachirops }\end{array}$ & $\begin{array}{c}\text { Moennigia } \\
\text { dessetae }\end{array}$ & Total \\
\hline I $\ldots \ldots \ldots \ldots \ldots \ldots$ & 17 & 3 & & 3 & 23 \\
II $\ldots \ldots \ldots \ldots \ldots \ldots$ & 20 & 5 & 3 & & 28 \\
III $\ldots \ldots \ldots \ldots \ldots$ & 13 & 3 & & 3 & 67 \\
Total $\ldots \ldots \ldots \ldots \ldots$ & 50 & 11 & 3 & 2,01 & \\
Pourcentages $\ldots \ldots$ & 33,5 & 7,37 & 2,01 & & \\
\hline
\end{tabular}

\section{Comparaison entre les faunes des Didelphis et du Metachirops :}

A l'analyse, la composition des deux faunes apparaît comme étant très proche. Sur les quatre genres connus à la fois chez Didelphis et Metachirops, trois sont communs ; Viannaia, Travassostrongylus, Moennigia et sur les huit espèces décrites, deux sont communes : $V$. viannai et $M$. dessetae. Chez les deux hôtes, $V$. viannai a la même localisation et est répartie de façon homogène ; $M$. dessetae est peu abondante et à localisation antérieure.

Morphologiquement nous avons vu (cf. Diaw, 1976) que $T$. paraquintus, parasite du Metachirops, était l'espèce la plus proche de $T$. tourei (parasite d'un des deux 
Didelphis). Les deux espèces, réparties dans tout l'intestin (sauf le quart postérieur pour T. tourei) pourraient donc être considérées comme des vicariantes.

Enfin $V$. metachirops et $H$. cayennensis, bien qu'appartenant à deux genres différents, sont deux espèces morphologiquement très atypiques, rares et à localisation bien précise : $V$. metachirops dans la partie moyenne et $H$. cayennensis dans la partie posté-

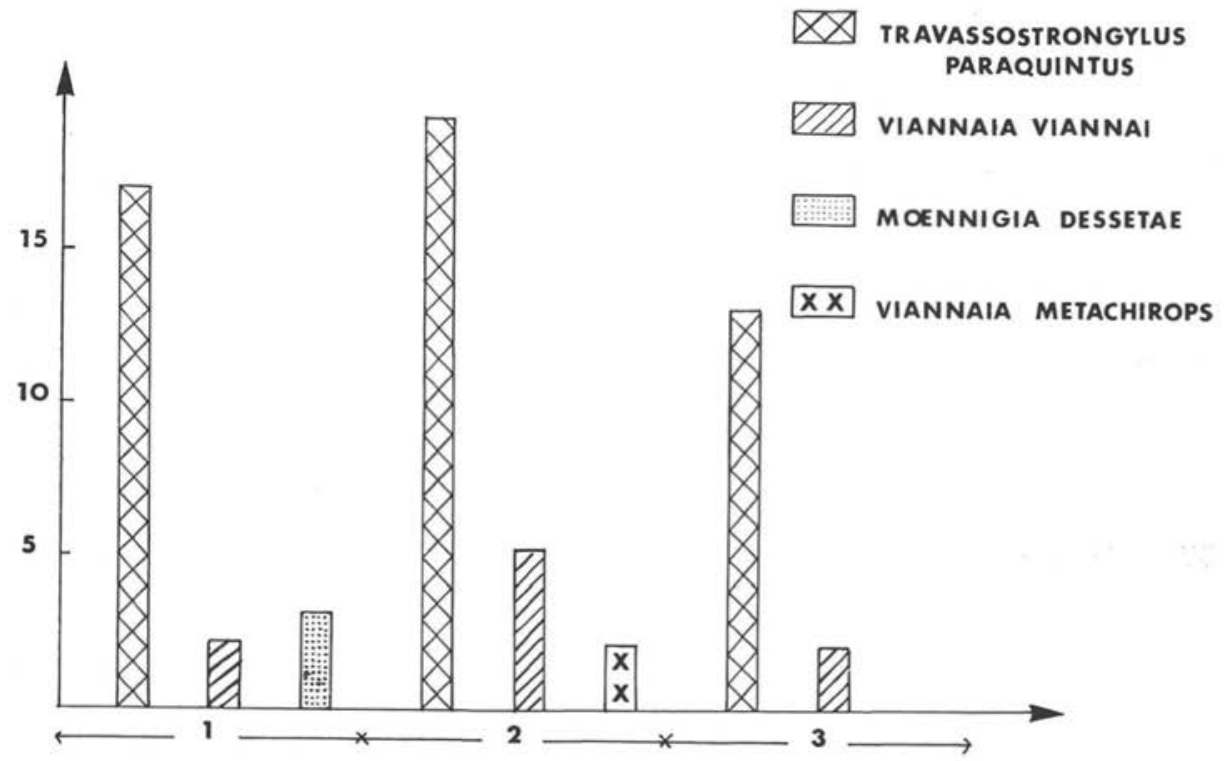

FIG. 3. - Répartition des différentes espèces de Trichostrongyloidea dans l'intestin d'un Metachirops opossum originaire de Guyane française (d'après Durette-Desset, 1974). L'intestin est divisé en trois segments. Les chiffres en abscisse indiquent le numéro du segment depuis l'estomac jusqu'au cæcum. La totalité des individus de chaque espèce trouvée dans chaque segment est portée en ordonnée.

rieure de l'intestin. Seules, $T$. callis et $T$. orloffi, présentes chez les deux Didelphis, n'ont pas leur équivalent chez le Metachirops.

Nous constatons donc que les deux faunes ont un équilibre général assez comparable. Il existe chez les deux faunes une espèce dominante, mais différente pour chaque hôte : $T$. paraquintus chez le Metachirops et $V$. viannai chez les deux Didelphis. De plus, alors que chez le Metachirops le nombre global de spécimens dans chaque partie de l'intestin est équivalent, il décroît d'avant en arrière chez les deux Didelphis. Nous pensons cependant qu'il ne faut attribuer qu'une valeur relative à ce résultat, car il porte seulement sur l'autopsie de trois animaux. 


\section{Hypothèse sur la formation de l'helminthofaune (Trichostrongyloidea)}

\section{chez les Marsupiaux néotropicaux}

Cette faune est composée de quatre genres: Travassostrongylus, Moennigia, Hoineffia et Viannaia. D'après Durette-Desset, 1971, les genres Moennigia, Viannaia et Travassostrongylus se répartissent en trois lignées : «Moennigia », «Viannaia », "Vexillata $»$ et en deux familles différentes: Heligmosomidae et Trichostrongylidae. Alors que Viannaia et Travassostrongylus sont des genres qui ont évolué chez les Marsupiaux, le genre Moennigia n'a réussi sa poussée évolutive que chez les Edentés chez lequels il existe un très grand nombre d'espèces. De plus, aussi bien chez Didelphis que chez Metachirops, c'est la même espèce ( $M$. dessetae) qui a été trouvée et en petit nombre. Nous considérons donc que la présence de Moennigia n'est pas primitive chez les Marsupiaux et nous pensons qu'il s'agit d'un parasite de capture. En ce qui concerne les autres genres, deux éléments semblent indiquer qu'on est en présence, en fait, d'une seule et même lignée évolutive :

1. Le synlophe du genre Viannaia est constitué de trois arêtes cuticulaires ventrales, Or, le synlope de la L 4 de Travassostrongylus est très proche de celui du genre Viannaia avec 6 arêtes ventrales dont 3 fortes.

2. Le genre Hoineffia possède une bourse caudale typique d'un Travassostrongylus primitif (côte 4 plus courte que la côte 5) et un synlophe typique du genre Viannaia.

L'hypothèse la plus vraisemblable est de considérer que les genres Travassostrongylus et Viannaia ont un ancêtre commun proche du genre Hoineffia (synlophe avec 3 arêtes ventrales, bourse caudale de Travassostrongylus primitif), et dont la femelle serait encore didelphe.

L'évolution se serait effectuée par la perte d'un utérus pour les genres Hoineffia et Viannaia, et par l'apparition d'arêtes dorsales pour le genre Travassostrongylus.

Contrairement aux apparences, nous pouvons donc considérer que les trois genres constituant l'helminthofaune de ces Marsupiaux appartiennent en fait à la même lignée évolutive.

\section{Conclusions}

D'après ce qui précède, l'helminthofaune des Marsupiaux est caractérisée par les éléments suivants :

1) L'abondance relative de chaque espèce est très variable d'un individu hôte à un autre, mais, cependant, dans les trois cas, nous avons trouvé une espèce dominante (Viannaia chez les Didelphis, Travassostrongylus chez les Metachirops). 
2) Du point de vue phylétique, excepté le genre Moennigia que nous interprétons comme un parasite de capture à partir des Xénarthres, il nous paraît possible de considérer que les genres Travassostrongylus, Hoineffia et Viannaia appartiennent à la même lignée évolutive. Ce sont les mêmes résultats qui ont été mis en évidence chez les Hystricidés. Ainsi, les parasites de l'Arthérure (Hystricidé africain) et du Trichys (Hystricidé asiatique) appartiennent à trois genres de la même lignée évolutive (cf. Durette-Desset, Diaw et Krishnasamy, 1975).

\section{Bibliographie}

Dııw (O.-T.), 1976. - Contribution à l'étude de Trichostrongyloidea parasites de Xenarthre, Marsupiaux et Rongeurs néo-tropicaux. Bull. Mus. nat. Hist. nat. (à paraître).

Durette-Desset (M.-C.), 1971. - Essai de classification des Nématodes Héligmosomes. Corrélations avec la paléobiogéographie des hôtes. Mém. Mus. nat. Hist. nat., nouv. sér., sér. A, Zool., 49, 126 p.

Duretre-Desset (M.-C.), 1974. - Nématodes (Trichostrongyloidea) parasites d'un Marsupial de Guyane. Ann. Parasit. hum. comp., 49, 555-566.

Durette-Desset (M.-C.), Diaw (O.-T.) et Krishinasamy (M.), 1975. - Quatre nouvelles espèces de Nématodes Héligmosomes parasites de l'intestin d'un Trichys lipura Günther malais; comparaison avec la faute d'Athérures congolais. Ann. Parasit. hum. comp., 50, 477-491.

Travassos (L.), 1914. - Trichostrongylinae brazileiros (Nota previa). Brazil Medico, 28, 163-164.

Travassos (L.), 1935. - Alguns novos generos e especies de Trichostrongylidae. Rev. Med. cirurgica do Brazil, 43, 345-361. 